\title{
Assessment of Scheduling and Plan Execution of Apollo 14 Lunar Surface Operations
}

\author{
Jessica J. Marquez* \\ NASA Ames Research Center, Moffett Field, CA 94035
}

\begin{abstract}
Although over forty years have passed since first landing on the Moon, there is not yet a comprehensive, quantitative assessment of Apollo extravehicular activities (EVAs). Quantitatively evaluating lunar EVAs will provide a better understanding of the challenges involved with surface operations. This first evaluation of a surface EVA centers on comparing the planned and the as-ran timeline, specifically collecting data on discrepancies between durations that were estimated versus executed. Differences were summarized by task categories in order to gain insight as to the type of surface operation activities that were most challenging. One Apollo 14 EVA was assessed utilizing the described methodology. Selected metrics and task categorizations were effective, and limitations to this process were identified.
\end{abstract}

\section{Nomenclature}

ALSEP $=$ Apollo Lunar Surface Experiment Package

$C D R=$ Commander

$E V A=$ extravehicular activity

ISS $\quad=$ International Space Station

$L M P \quad=$ Lunar Module Pilot

$M E T \quad=$ mobile equipment transporter

\section{Introduction}

$\mathrm{P}$ lanning and re-planning human planetary surface operations is a complex and safety critical task. Understanding and assessing how surface operations were planned and executed during Apollo will aid in the development of future planetary surface operational concepts. It will contribute to our understanding of the challenges of planetary surface operations, leading to better extravehicular (EVA) surface operations modeling and planning. In order to evaluate Apollo surface operations, the following focused research is proposed: to quantitatively assess lunar EVAs, within and across missions, in order to determine what were the most challenging aspects of completing EVAs. This will be accomplished by comparing planned timelines for each Apollo EVA with the as-run (i.e., executed) timeline. Such an assessment should be comprehensive, across all missions and EVAs. The initial step is to determine the appropriate assessment method to apply to all lunar EVAs. This paper describes a possible method using one of the EVAs conducted during Apollo 14.

\section{Background}

Several assessments of extravehicular activities have been published, and are subsequently reviewed. Current research described in this paper differs and complements existing work as it leverages published EVA assessment methods yet focuses on planned and executed timelines.

\section{A. Apollo Reviews}

During the Apollo era, some assessments of Apollo EVAs were published, most as internal reports. Many of these reports were conducted in a compressed timeframe since all six Apollo missions that landed on the Moon occurred within a three-year time frame. Each Apollo mission was reviewed and qualitative assessments were published in the individual Technical Debriefs and Mission Reports. Complementary to these reports is work by

\footnotetext{
* Human Systems Integration Division, NASA Ames Research Center, M/S 262-2, Moffett Field, CA 94035, and
} AIAA Senior Member. 
Marquez and Newman ${ }^{1}$, which reviewed the sections pertaining to surface operations (i.e., EVAs) in order to develop a generalized overview of key factors that influenced EVA re-planning. Yet to date, there is no comprehensive, quantitative evaluation of all Apollo EVAs.

Some Apollo missions received additional attention. For instance, Waligora and Horrigan ${ }^{2}$ published data and assessment for Apollo 14, EVA 2. This particular EVA happens to be to the longest walked traversal accomplished during Apollo; hence, it was also one of the most physically demanding EVAs. Additionally, the crew encountered navigation and situation awareness issues that hindered their ability to execute their traversal as planned. Carr et al ${ }^{3}$ elaborated on the data published from that EVA in order to conduct a more extensive, quantitative evaluation of metabolic costs and energy expenditure modeling. One other noteworthy memorandum, written just before the launch of Apollo 14, suggests methods of organizing lunar surface operations timelines ${ }^{4}$. Slaybaugh organizes the Lunar Surface Procedures into "a quantitative, semi-detailed listing of EVA activity times and sequences" which the author recommends as a way to evaluate time and energy expenditure uncertainties. Also, the authors attempted to summarize activities into a simple categorization: housekeeping, science (ALSEP, sample, other), describe/photo, travel, and other.

Apollo 15 and 16 have time-and-motion studies that were reported soon after their completion ${ }^{5,6}$. While these reports appear to emphasize locomotion (e.g., comparing locomotion data such as speed and metabolic rates within EVAs), they do provide some quantitative evaluation of tasks conducted on the surface. For example, energy expenditures as a function of completed tasks are assessed. The authors also compare times to complete tasks between training and executed EVAs. They leverage common tasks across the missions (e.g., deploying the same experiment) and assess time to complete task differences. This is the only published reference found that attempts to assess EVA task efficiencies across missions.

\section{B. Current EVA Reviews}

Reviewing previous Apollo EVA reports highlighted some key elements for quantitative evaluation: listings of EVA activity times, groupings of activities, and time comparisons. More recent work on microgravity EVAs presents further refinement on these evaluation elements. Looper and Ney have started to address EVA task efficiencies for microgravity spacewalks ${ }^{7-10}$. Based on their extensive experience with EVA operations, the authors propose four major categories which EVA tasks can be classified: work objective, support equipment, translation, and worksite preparation ${ }^{7}$. They then applied this categorization to tasks performed in International Space Station (ISS) Increment 9 EVA and Earth-analog EVAs (for instance, Antarctic expedition). With respect to ISS tasks, data was collected by monitoring space-to-ground voice loops and manually time-stamping procedure steps. As a result, an EVA can be described with percent time spent on each of the task categories.

In subsequent assessments ${ }^{8-10}$, additional categories have been added in order to accommodate different types of EVAs. For instance, when assessing Russian and U.S. EVAs, the authors had to include categories for troubleshooting ${ }^{8-9}$ and rest $^{8}$. Their evaluation of Earth-analog EVAs further expanded the nomenclature to include robotics ${ }^{10}$. While allowing for supplementary categories, Looper and Ney provide a method for assessing EVA task distributions and quantification of tasks across EVA instances.

It is important to note their method for data collection. Their preferred technique for data collection relies on following along voice communications, tagging individual procedure steps (with a resolution of one minute), and binning these steps into the appropriate categories. One potential shortcoming of this process is ensuring consistent categorization of procedure steps. The authors do note that it could be challenging to inarguably categorize each task, and subsequently, the corresponding time on task data.

\section{Methodology}

Based on the review of previous lunar and microgravity EVA assessments, an amalgamated method for quantitatively evaluating Apollo surface operations was determined. This method leverages Looper \& Ney's data collection and assessment techniques, and adapting it for lunar EVAs timeline comparisons. The methodology for quantitatively assessing lunar surface operations relies on data collection of both 1) planned and 2) executed activities for each EVA, assembling sequence and durations for each activity. Next, timelines are compared and discrepancies summarized using the differences (between planned and executed) in task durations as a quantitative measure.

\section{A. Data Collection: Planned Tasks}

In order to assess Apollo EVAs, data on EVA activities have to be collected and organized. For this paper, multiple sources were used, as a single source was not sufficient to extract all needed data. This phase of the 
evaluation process is time consuming as sources (e.g., reports, transcripts, recordings) had to be distilled and data had to be manually digitized.

Each Apollo mission has a published "Final Lunar Surface Procedures" report that contains detailed descriptions of planned surface operations. This was the main source of information for planned task data. Among the information found in this document are associated goals and images of experiments, EVA timelines, EVA cuff checklists, and planned contingency procedures. Of particular interest was the timelines, which listed activities with corresponding estimated times for each crewmember. Each timeline provided a set of tasks that were already agreed upon by the EVA planners. Additionally, the cuff checklists contained more precise information of associated times for procedures that had to be executed during an EVA (Figure 1). Timelines and cuff checklists were used to generate a list of activities to be performed by each crewmember (the Commander, CDR, and the Lunar Module Pilot, LMP), and the expected start and end times for each of these activities (and hence, estimated task duration). Tasks that required coordination between CDR and LMP were noted. All activities followed in sequence; in other words, the end time of one activity was also the

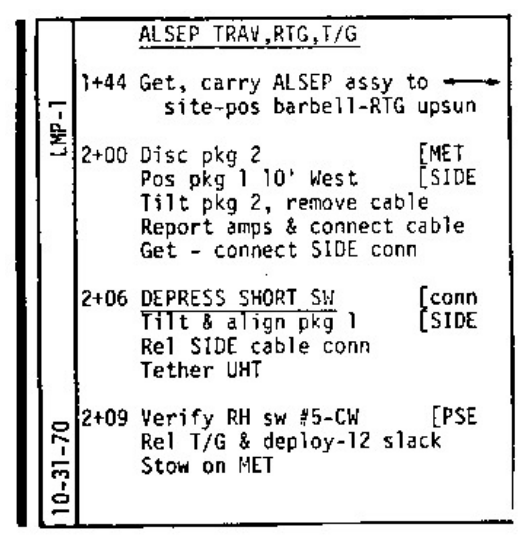

Figure 1. Example of an Apollo 14 EVA cuff checklist.

start time for the next activity. Slaybaugh similarly used Apollo 14's Final Lunar Surface Procedures ${ }^{11}$ to organize tasks with corresponding start times and durations, which was referenced as an EVA Activities Sequence Chart ${ }^{4}$.

A limitation of this planned tasks data collection method is that not all activities have clear start and end times. The cuff checklists and timelines provided were used to estimate appropriate times. Occasionally, specific procedures were more suitable to use as a task since they provided a more appropriate resolution. For instance, a task that was labeled "Procedure A \& B" was broken into two tasks, "Procedure A" and "Procedure B".

\section{B. Data Collection: Executed Tasks}

Gathering and organizing the data of actual start times and durations of Apollo EVA tasks as executed requires various sources. As mentioned, Apollo 15 and 16 have time-and-motion studies ${ }^{5-6}$ but these are not comprehensive for all EVA tasks and every Apollo mission. In another NASA report, Orloff provides a time stamp for steps conducted for each Apollo mission, i.e., "as-run" timeline for the entire mission". These timelines for each EVA (for each mission) can be extracted. Unfortunately, the steps listed in these "as-run" timelines do not always match the nomenclature established by planned timelines. On occasion, multiple tasks are grouped and tasks are not necessarily separated by crewmember. However, they do provide ballpark estimates of start times.

Using these estimates, voice communication transcripts between ground and crewmembers were used to ascertain executed start and end times of planned tasks. Time-stamped transcriptions of Apollo voice communications are available through the Apollo Lunar Surface Journal, which are further annotated with crew interviews, reports, images, and video \& voice recordings ${ }^{12}$. Actual start and end times for planned tasks were determined, cross-referenced with planned timelines, EVA cuff checklists, and the crewmember verbal confirmations of tasks in voice transcripts. This method, akin to Looper and Ney ${ }^{7}$, allowed for task durations to be gathered, as well as to establish if new tasks were inserted or if tasks were rearranged as compared to the planned timeline. In addition, reading and listening to the voice transcripts provided much insight as to reasons behind delays, challenging tasks, and other circumstantial activities that may have affected the execution of planned tasks. These notes were captured alongside tasks for future reference.

\section{Analyzing Apollo 14}

Before collecting data for all surface EVAs conducted in each Apollo mission, one mission was initially evaluated in order to test out the determined methodology. Apollo 14 (Figure 2) was selected as a candidate for several reasons: for its various scientific experiments, geological excursions, and lack of Lunar Rover Vehicle. Selecting a mission without the rover was deemed a "simpler" EVA operational environment. In addition, the EVAs in Apollo 14 were known to have necessitated replanning ${ }^{1-3}$. This was essential as the focus of this research is to assesses the surface operational challenges, comparing planned versus as-run EVAs. 
Collecting data for the planned tasks and associated time for each task was completed for both of the Apollo 14 EVAs. EVA 1 was a relatively straightforward traversal, where the astronauts deployed scientific instruments and did not stray far from the lunar module. Its timeline was reasonably well defined and procedure steps demarked task start times. Identifying the as-run timeline required extensive cross-referencing with the voice transcripts, and only occasionally did start times have to be estimated (i.e., there was no specific announcement by $\mathrm{crew}^{\dagger}$ ).

On the other hand, EVA 2 was more focused on geological examination of the area, and large portions of the timeline were marked under one task: geological traverse. There was not enough resolution on the listed planned tasks though several intermediate waypoints and their time to traverse to each was estimated by Apollo mission planners. As documented ${ }^{1-3}$, there were navigation and situation awareness issues that drove crewmembers to adapt the traversal, creating a very different as-run timeline. Subsequently, it became apparent that analyzing EVA 2 with respect to comparing planned and as-run timelines would not be effective if attempting to initially test the determined evaluation method for surface EVAs. Hence, the focus of this paper is EVA 1, because the planned and as-run timelines could be parsed and compared more readily.

\section{Categorizing Tasks \& Preliminary Set of Metrics}

The aim of this assessment is to understand what assigned surface tasks were operationally difficult to perform as surmised by their execution during Apollo EVAs. This can be achieved by comparing planned and executed timelines as a whole. However, summary information is necessary to evaluate across missions and EVAs, increasing the amount of data collected, and hence, provide better insight to which types of tasks affected surface operations the most. Looper and $\mathrm{Ney}^{7}$ provide EVA characterization categories; this paper leverages these and adapts the category definitions to suit planetary EVAs (Table 1).

Table 1. EVA Task Categories

\begin{tabular}{|l|l|l|}
\hline \multicolumn{1}{|c|}{$\begin{array}{c}\text { Looper \& Ney } \\
\text { Categories }\end{array}$} & $\begin{array}{c}\text { Adapted Definition for Planetary Surface Operation } \\
\text { Timelines }\end{array}$ & \multicolumn{1}{c|}{ Adapted Categories } \\
\hline Work Objectives & $\begin{array}{l}\text { Tasks associated with deploying tools, scientific or } \\
\text { otherwise, enabling them, and/or collecting } \\
\text { samples. }\end{array}$ & Science Task \\
\cline { 2 - 3 } Support Equipment & $\begin{array}{l}\text { Tasks associated with offloading or preparing tools, } \\
\text { be it science or operational equipment. }\end{array}$ & Science-related Setup \\
\cline { 3 - 4 } Worksite Preparation & $\begin{array}{l}\text { Tasks not associated with work objectives but that } \\
\text { support conducting a surface EVA. }\end{array}$ & Operations-related Setup \\
\hline EVA Translation & Tasks associated with moving across the surface. & Traverse \\
\hline
\end{tabular}

While the Looper and Ney categories are appropriate, a few subcategories are identified to investigate if there is a distinction between these types of tasks. Work objective tasks are broken down into two types: science and operations tasks. Likewise, setup for these tasks are also separated. Compounding the issue, however, is the fact that some tasks served both science and operational objectives (for example, taking pictures of a deployed instrument). Ambiguity in categorization is resolved by deciding if the task enabled science or operations more. Future adaptation may include subcategorizing EVA translation to distinguish between walking and using the rover. Because the adapted categories (with some renaming) have a direct parallel to the categories proposed by Looper \&

\footnotetext{
${ }^{\dagger}$ According to Jones, the crews of subsequent Apollo missions were more verbose ${ }^{12}$.
} 
Ney, both categories were used in this analysis in order to explore the possibility of teasing out distinctions between science- and operational-related tasks.

The basic unit of measurement is time, be it time to complete a task or many tasks, bounded by a start and end times. Using this variable, other metrics were calculated:

- Percent distribution of time spent in each EVA category (akin to Ref. 7); and

- Time spent on task normalized to planned time, averaged over each EVA category.

\section{EVA Timeline Assessment}

\section{A. Comparison of Planned vs. As-Run Timelines}

Figure 3 summarizes the planned EVA 1 timeline for Apollo 14 and the corresponding as-run timeline. During EVA 1, the commander (CDR) and lunar module pilot (LMP) had to complete a list of tasks with the mobile equipment transporter (MET) in approximately 4 hours and 15 minutes. Among operational tasks was deploying a TV camera, erecting the S-band antenna, and displaying the American flag. Among science tasks was deploying the Apollo lunar surface experiments package (ALSEP, which had six experiments), and collecting and geologically documenting the area via photographs and samples. The crew did not have to traverse far from the Lunar Module (i.e., lander) in order to complete their tasks, which were divided between the crewmembers. A few tasks required coordination, such as transferring items between the surface and the lander. Ultimately, all major tasks were achieved but the EVA overran by about 30 minutes ( $13 \%$ over the total time).
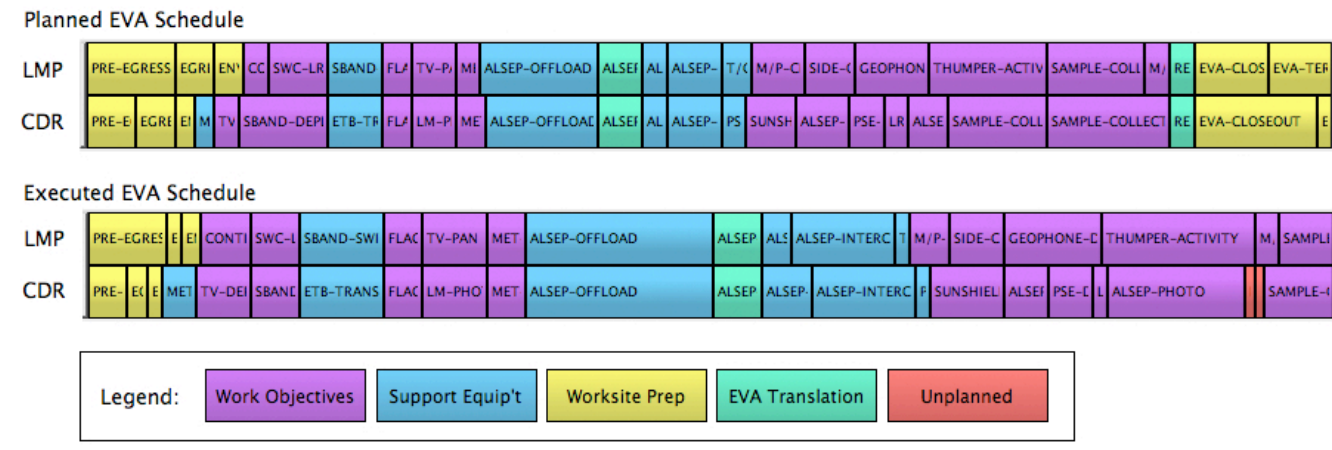

Figure 3. Summary of planned and as-run EVA 1 (Apollo 14) broken down by crewmember and colored by Looper \& Ney EVA categories.

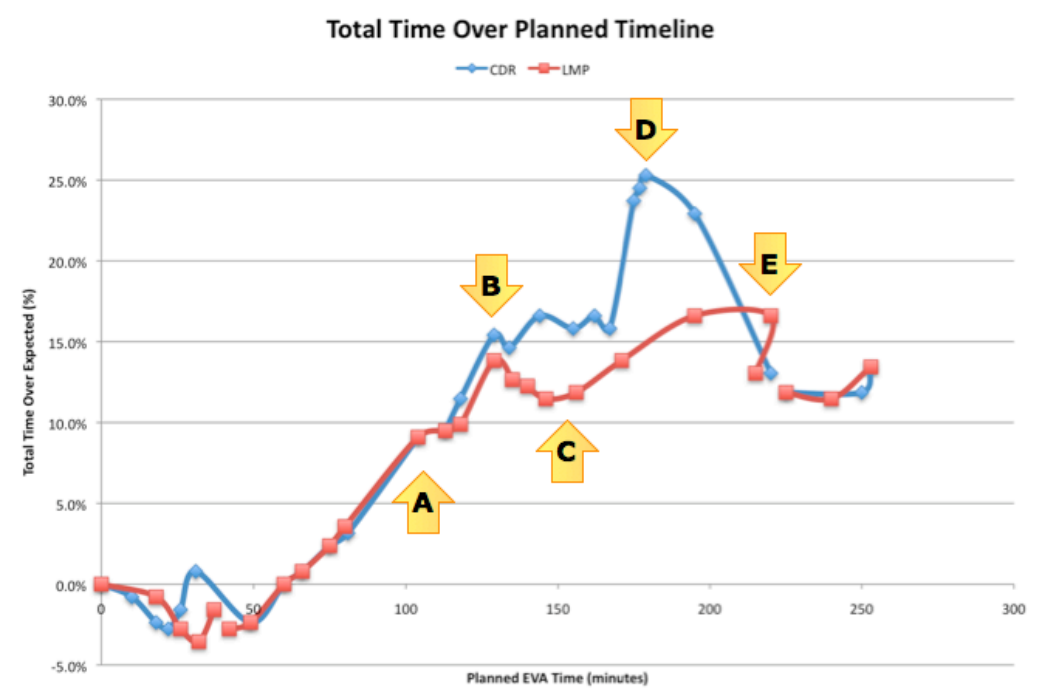

Figure 4. Percent over total expected time as a function of planned EVA 1 time (Apollo 14). Labeled are notable EVA events that contributed to the total time overrun.

American Institute of Aeronautics and Astronautics 
A first-order assessment of which activities took longer to accomplish than expected was prepared by plotting planned EVA time versus the total time over schedule at that point in time (Figure 4). This plot identifies some interesting trends. Early in the EVA, the crew was either ahead of the planned schedule or right on time. After the 50-minute mark, the crew steadily takes longer to complete tasks, adding to the total overrun time for the EVA. The following events were recognized as notably affecting the execution of the EVA (in reference to Figure 4):

A. Completing "ALSEP Offload" doubled the percent time over expected timeline;

B. Completing "ALSEP System Interconnect" increased the percent time over expected about another 50\%;

C. While LMP managed to buy back some time while deploying several experiments, the CDR did not;

D. Completing "ALSEP Photography" by CDR increased the percent time over expected about another 50\%.

E. Re-planning occurred: LMP reordered activities and geological traverse activity was removed.

Even though overall, EVA 1 ran about 30 minutes over schedule, this was only $13 \%$ of the overall time allotted for the EVA. Several different aspects of setting up the ALSEP experiments appear to be the largest contributors to time delays when comparing planned versus executed timeline tasks. Changes to the timeline, i.e., re-planning, took effect about 3 hours, 40 minutes into the EVA. However, the CDR was at one point $25 \%$ over his planned EVA time.

Relative to each task, the average percent time over was larger for each task. Percent time over was calculated by determining the time duration differences between expected and executed, normalized to planned duration. Table 2 summarizes the average percentages and the differences between CDR and LMP. Figure 5 depicts the distribution of percent time over per task as a function of crewmember. While the data (compromised of 49 total examples) appear to be normally distributed, they are not ( $\mathrm{p}<0.01$, Shapiro-Wilk test). Arguably, the limited data may not be enough to determine normality, however, for this EVA, the activity "ALSEP Photography" seems to have skewed the distribution. This activity was about ten times the average percent time over per task. Statistical comparisons between the average percent time over by crewmembers are limited, not just because of the limited number of data points, but also because the data do not meet many of the statistical test assumptions ${ }^{\ddagger}$.

Table 2. Descriptive statistics for percent time over per task, by crewmember

\begin{tabular}{|c|l|c|c|c|}
\hline \multicolumn{2}{|c|}{} & Number & Mean & $\begin{array}{c}\text { Standard } \\
\text { Deviation }\end{array}$ \\
\hline \multirow{3}{*}{ Percent Time Over } & CDR & 24 & $31.8 \%$ & $73.1 \%$ \\
\cline { 2 - 5 } & LMP & 25 & $15.2 \%$ & $50.2 \%$ \\
\cline { 2 - 5 } & Total & 49 & $23.3 \%$ & $62.4 \%$ \\
\hline
\end{tabular}

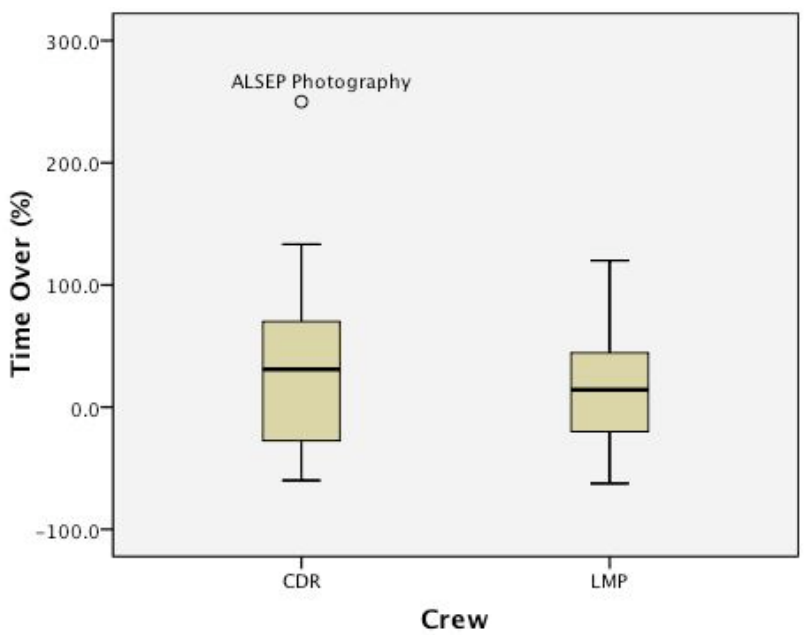

(a)

\begin{abstract}
Crew
\end{abstract}
(b)

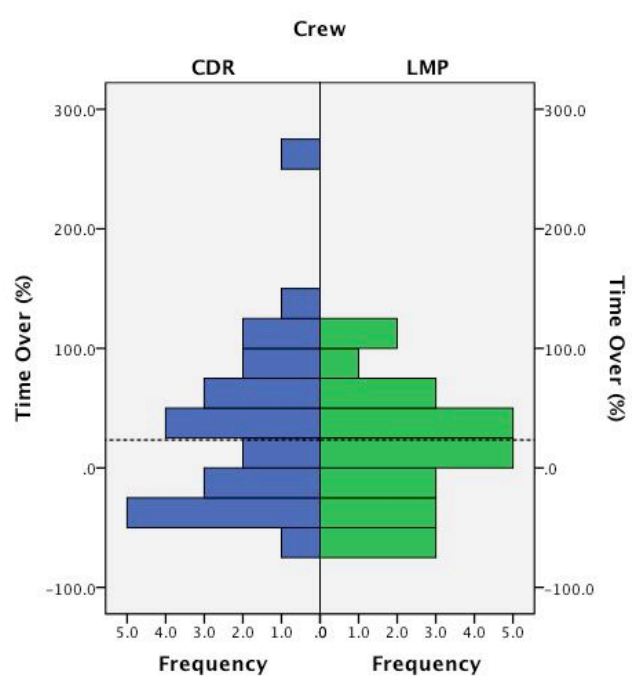

Frequency Frequency

Figure 5. Percent time over per task by crewmember in boxplots (a) and histogram (b).

\footnotetext{
\$Specifically, t-tests are inappropriate (the data are not normally distributed) and non-parametric tests assume either the data are independently sample (but several activities were coordinated) or dependent (yet crew had different tasks).
} 


\section{B. Categorization of EVA Activities}

As previously mentioned, two types of categorizations were utilized to group activities for this EVA: Looper \& Ney's categories and adapted categories. The portion of the total time spent on each task category are shown in Figure 6 with Looper \& Ney's categories and in Figure 7 with adapted categories. The charts include planned and as-executed percentages. These percentages show that for EVA 1, most of the time should have been spent on work objectives activities and roughly equal time should have been spent between support equipment and worksite preparation tasks. The as-run chart reveals that a greater percentage of time was spent on support equipment tasks than expected, and the crew had to compensate for this by eliminating much of the time spent translating about the lunar surface. Only a very small percentage of time was spent on unplanned activities. The charts with the adapted categories show that more time was to be devoted to science tasks and their setup, though when executed, the activities related to science setup were the ones that incurred the most time delays. This agrees with the previous identified tasks ("ALSEP Offload" and "ALSEP System Interconnect") that earned the highest time penalties; these were categorized as Science-related Setup tasks. While "ALSEP Photography" task duration was poorly estimated (as it had the highest percent total time over), its overall effect was relatively small as it (along with all science task delays) only added another $2 \%$ of total time.

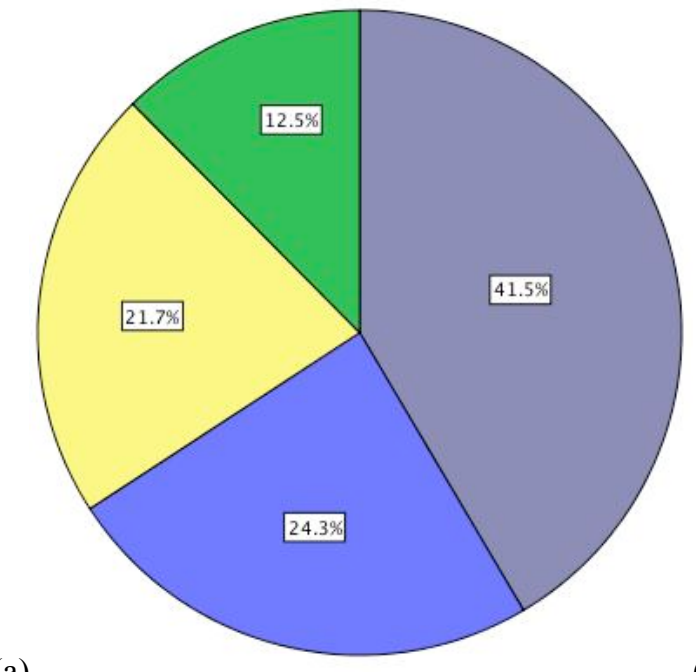

(a) executed (b).

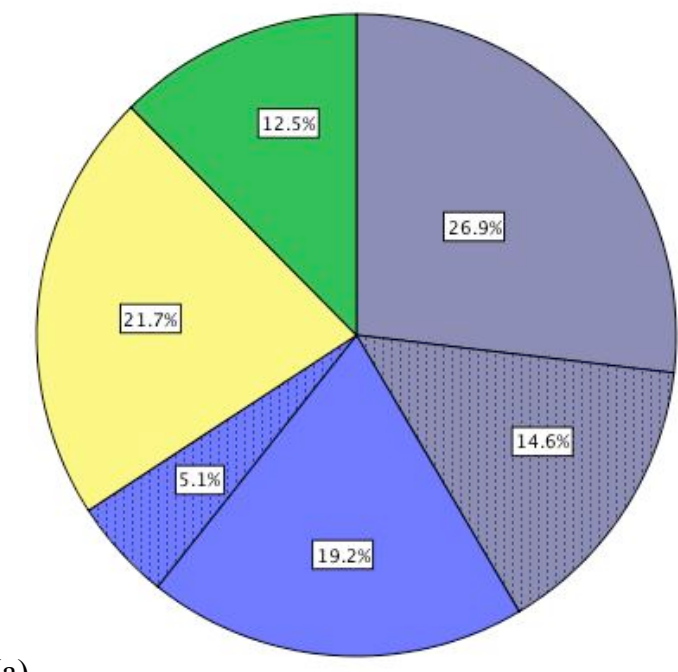

(a)

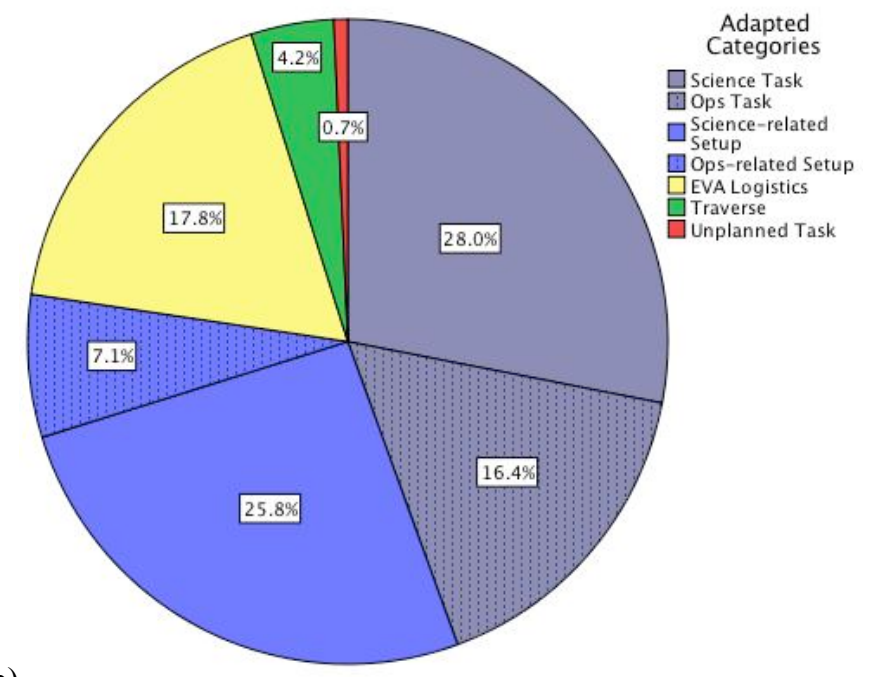

(b)

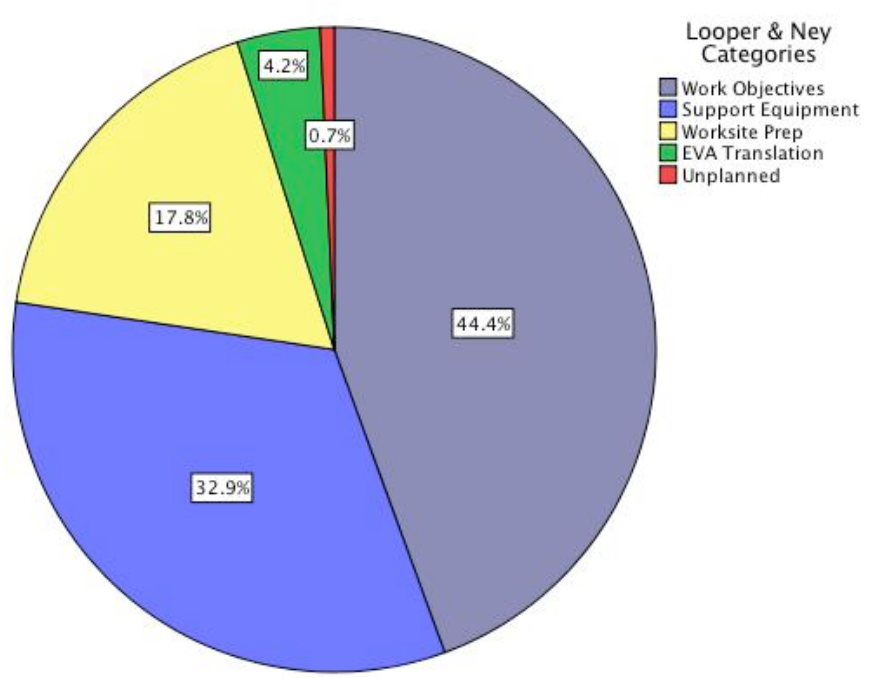

(b)

(a) and as

Figure 7. Percent of total time spent per adapted EVA activity categories, as planned (a) and as executed (b). 
Average percent time over per EVA task category may provide insight as to which type of tasks took longer than expected, summarized in Table 3. Unfortunately, due to the nature of the data and the number of data points, comparing means is not feasible. Figure 8 (boxplots with medians and range of data) illustrates that there were more time delays associated with work objective and support equipment tasks than worksite preparation and EVA translation tasks - which actually had negative mean percent times over. Negative means (Table 3) could be interpreted as either tasks that were omitted or tasks that took less time to complete than expected. In the case of EVA translation tasks, these were omitted in order to compensate for delays. With respect to the adapted categories, average durations for operations tasks were longer than those for science tasks. However, the overruns on science tasks covered a wider range - some science tasks were completed early or took a long time.

Table 3. Descriptive statistics for percent time over per EVA categories

\begin{tabular}{|c|c|c|c|}
\hline EVA Categories & Number & Mean & Std. Deviation \\
\hline Work Objectives & 23 & $32.3 \%$ & $68.6 \%$ \\
\hline - Science Tasks & 14 & $26.4 \%$ & $82.3 \%$ \\
\hline - Operations Tasks & 9 & $41.6 \%$ & $42.1 \%$ \\
\hline Support Equipment & 12 & $44.5 \%$ & $49.3 \%$ \\
\hline - Science-related Setup & 9 & $38.9 \%$ & $56.2 \%$ \\
\hline - Operations-related Setup & 3 & $61.4 \%$ & $11.8 \%$ \\
\hline EVA Translation/Traverse & 4 & $-24.4 \%$ & $41.1 \%$ \\
\hline Worksite Prep/EVA Logistics & 10 & $-3.7 \%$ & $55.6 \%$ \\
\hline Total & 49 & $23.3 \%$ & $62.4 \%$ \\
\hline
\end{tabular}

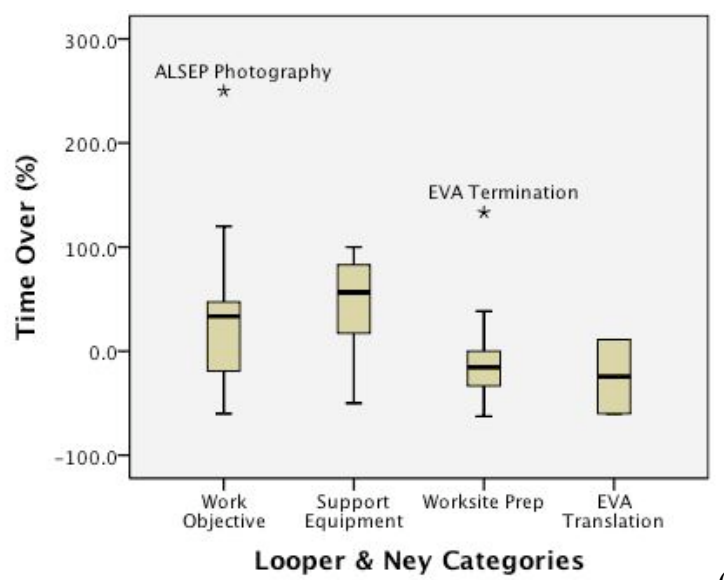

(a)

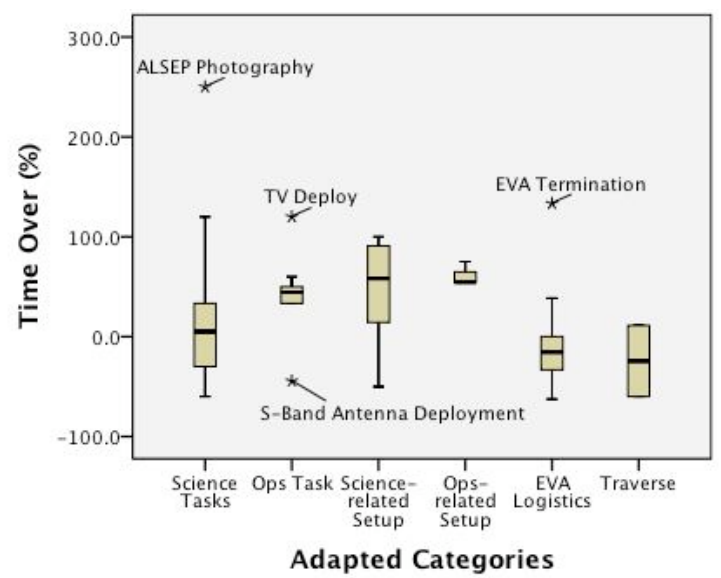

Adapted Categories

Figure 8. Boxplot of percent time over by EVA categories, Looper \& Ney (a) and adapted (b).

Summarizing data with Looper and Ney EVA categorizations was useful, as it provided insight as to the type of activities in general that were challenging to execute within an EVA. For instance, support equipment tasks took a larger percentage of the overall EVA duration than expected. The adapted categories, on the other hand, appear to supply further details. The support equipment tasks that were related to science tasks consumed more of their allotted time even though the tasks related to operations took longer to complete than expected. With both of these categorizations, there was not enough data to conduct statistical analysis across the categories.

\section{Assessment Summary}

After Apollo 14, the crew agreed that there were aspects of the EVA that were challenging and not enough time was planned for:

"Timeline: operations on the lunar surface required a much longer time than had been anticipated. The planned activities required 25 to 30 percent more than would be required under one-g conditions. Scheduling additional activities, in the event that certain portions of the extravehicular activity have to be cancelled, is advisable."13 
With respect to the first EVA, this estimate is relatively close to the average over all the tasks $(23 \%)$ and work objective activities (32\%). Unfortunately, the tasks that related to support equipment (setting up science- or operational-related equipment) took on average longer $(45 \%)$ and consumed a larger percentage of time for the EVA than expected. In order to compensate for these overruns, the crew had to diminish the amount of time spent traversing. Ironically, the second EVA included a lot of traversals.

While this particular crew estimates that $25-30 \%$ padding on the tasks was desirable, this was based on their own experience and the particular EVAs they were asked to complete. A summary of Apollo operational considerations ${ }^{14}$ on the other hand recommends $50 \%$ padding. According to Looper and $\mathrm{Ney}^{8}$, Shuttle adds an additional $20 \%$ to EVA timelines while ISS spacewalks, 50\%. Potentially, padding recommendations for planetary EVAs could be based on the type of tasks, though more assessment is required.

For this Apollo 14 EVA 1, the activities that appear to have contributed the most to timeline delays were associated with the ALSEP. ALSEP was critical to having a successful EVA mission, and hence, it had to be completed. Deploying the different experiments that were contained in this package did not go as expected, and thus took much longer time, as confirmed by the mission voice transcripts. There were various reasons for why tasks took longer to complete than expected. For instance, crew had to unexpectedly deal with undoing stuck bolts that held experiments into the package or finding an appropriate place to deploy the ALSEP experiment package (poor estimation of terrain).

Interestingly, while the assessment indicates the task that unexpectedly took the longest to complete was "ALSEP Photography", there is nothing in this evaluation that would indicate why this straightforward task would take so long relative to other work objective activities. The reason behind this delay is gleaned from the voice transcripts. During the EVA, the CDR had to complete the "ALSEP Photography" at the same time as the LMP was completing "Thumper Activity". The photography activity was not originally scheduled to occur at the same time (see Figure 3). Unfortunately, the "Thumper Activity" (part of seismic experiment) required that the crewmembers be still for a short period of time every time the thumper was activated (Figure 9). As a result, the CDR had to pause and coordinate with the LMP continuously throughout these activities. The final effect was that CDR took two and a half times longer to take photographs, adding time to an already delayed timeline execution. Capturing the effects of coordinated tasks, planned or unexpected, appears to be a limitation on the currently selected assessment method.

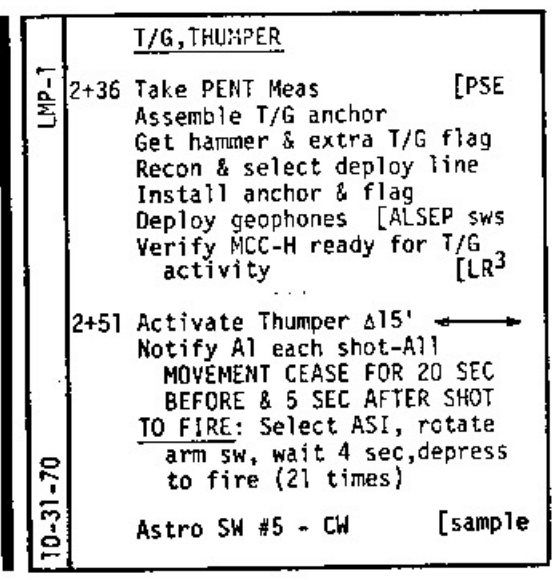

Figure 9. Cuff checklist procedure detailing LMP's "Thumper Activity"

\section{Conclusion}

A quantitative method for evaluating Apollo EVAs was amalgamated from previous and current EVA assessments that leverage planned and as-run timelines. This method was applied initially to an Apollo 14 EVA. From the evaluation, the following metrics could be gathered: total additional time spent on EVA, on each task, and types of types. Comparing averages across tasks and groups of tasks provides insight as to which surface operations tasks were challenging. Due to the limited amount of data points, statistical comparisons were not feasible. By far, the most demanding aspect of this assessment method is the collection of data, as it is time consuming. However, obtaining a comprehensive set of Apollo EVA task timing data may permit statistical comparisons across task categories, providing insight as to which type tasks contribute the most to re-planning.

Future work will aim at further evaluating other Apollo EVAs, simpler ones and more complex ones, further testing the robustness and limitations of chosen methodology. One potential barrier for assessing all Apollo EVAs is that there may be different types of EVAs. This was already encountered when attempted to evaluate the second Apollo 14 EVA. This EVA may be categorized as complex, not because of the activities per se, but rather because of the issues encountered during execution. Potentially, the planned and executed timelines of EVAs with long traverse portions can be further parsed to include traverse segments to waypoints.

There are some identified limitations. In this evaluation, it was not possible to tease out the effect of coordinated tasks, planned or otherwise. Addressing this limitation is hindered by the fact that not many planned activities were coordinated. Another limitation is that individual crew skills will invariably affect any derived metric for EVA 
assessments. The Apollo astronauts' ability to complete assigned tasks was inherently tied to their own experiences and expertise. Finally, a third limitation, as previously mentioned, is that the researcher may introduce some error in the data collection as occasionally start and stop times of tasks had to be estimated and task categorization is subjective.

Studying Apollo 14 first EVA was completed in order to exercise a method of collecting and assessing all Apollo EVA data. While no EVA, be it lunar or in microgravity, is executed exactly as planned, comparing planned versus as-run timelines is valuable because it provides a consistent method of evaluating EVAs. The underlying goal is to quantitatively assess lunar EVAs within and across missions, and as a result, increase our understanding of the challenges that are inherent to planetary surface operations.

\section{References}

${ }^{1}$ Marquez, J.J. and Newman, D.J. "Mission Planning and Re-planning for Planetary Extravehicular Activities: Analysis of Excursion in a Mars-Analog Environment and Apollo." International Conference on Environmental Systems, Norfolk, VA, July 2006.

${ }^{2}$ Waligora, J.M. and Horrigan, D.J. "Chapter 4: Metabolism and Heat Dissipation during Apollo EVA Periods." In R.S. Johnston, L.F. Dietlein, and C.A. Berry (Eds.), Biomedical Results of Apollo. NASA SP-368. Washington, DC, 1975. Pg. 115128.

${ }^{3}$ Carr, C.E., Newman, D.J., and Hodges, K.V. "Geological Traverse Planning for Planetary EVA.” International Conference on Environmental Systems. Vancouver, BC, Canada, July 2003.

${ }^{4}$ Slaybaugh, J.C. "A Method of Depicting Lunar Surface EVA's, Illustrated for the Apollo 14 Mission.” Bellcomm, Inc. NASA-CR-116948, January 26, 1971.

${ }^{5}$ Kubis, J.F., Elrod, J.T., Rusnak, R., and Barnes, J.E. “Apollo 15 Time and Motion Study.” NASA Manned Spacecraft Center M72-4, January 1972.

${ }^{6}$ Kubis, J.F., Elrod, J.T., Rusnak, R., Barnes, J.E., and Saxon, S.C. "Apollo 16 Time and Motion Study." NASA Manned Spacecraft Center M72-6, July 1972.

${ }^{7}$ Looper, C. and Ney, Z. "Extravehicular Activity Task Work Efficiency." International Conference on Environmental Systems, Rome, Italy, July 2005.

${ }^{8}$ Looper, C. and Ney, Z. "Quantifying EVA Task Efficiency." SpaceOps 2006, Rome, Italy, June 19 - 23, 2006.

${ }^{9}$ Looper, C. and Ney, Z. "EVA Task Timing and Timeline Planning." International Conference on Environmental Systems, Chicago, IL, July 2007.

${ }^{10}$ Looper, C. and Looper, A. “Task Efficiency Results for Desert RATS 2007.” International Conference on Environmental Systems, San Francisco, CA, June 2008.

11، Apollo 14 Final Lunar Surface Procedures” NASA Manned Spacecraft Center, December 31, 1970.

${ }^{12}$ Jones, E.M. “Apollo Lunar Surface Journal.” [online], http://www.hq.nasa.gov/alsj/ [retrieved January 2010].

13،Apollo 14 Mission Report.” Manned Spacecraft Center MSC-04112. Houston, TX, 1971.

${ }^{14}$ Engle, M. "Operational Considerations for Manned Lunar Landing Missions - Lessons Learned from Apollo." Space 2004 Conference and Exhibit, San Diego, California, Sep. 28-30, 2004.

${ }^{15}$ Orloff, R.W. "Apollo by the Numbers: A Statistical Reference" NASA Headquarters, NASA SP-2000-4029. Washington, DC, 2000. 